\title{
An extremely rare phenomenon of mucinous cystic neoplasm of the liver: spontaneous rupture
}

\author{
Xiaofeng Xu ${ }^{1}$, Chuanhui Peng ${ }^{1}$, Rongliang Tong ${ }^{1}$, Miaoxia Dong ${ }^{2}$, Junfang Deng ${ }^{1}$ \\ ${ }^{1}$ Division of Hepatobiliary and Pancreatic Surgery, Department of Surgery, The First Affiliated Hospital, Zhejiang University School of Medicine, \\ Hangzhou, China; ${ }^{2}$ Sir Run Run Shaw Hospital, Zhejiang University School of Medicine, Hangzhou, China \\ Correspondence to: Junfang Deng, MD. Division of Hepatobiliary and Pancreatic Surgery, Department of Surgery, The First Affiliated Hospital, \\ Zhejiang University School of Medicine, No. 79 Qingchun Road, Hangzhou, China. Email: junfangdeng@zju.edu.cn.
}

Submitted Mar 08, 2021. Accepted for publication Apr 14, 2021.

doi: $10.21037 / \mathrm{hbsn}-21-102$

View this article at: http://dx.doi.org/10.21037/hbsn-21-102

Mucinous cystic neoplasm of the liver (MCN-L) are rare cyst-forming epithelial neoplasm of the liver named by World Health Organization in 2010 (1,2). Most of MCN-L patients have no obvious symptoms, some patients have some nonspecific symptoms such as abdominal distension or pain, nausea, and vomiting (3). MCN-L patients rarely present with obstructive jaundice, cholangitis, intra-cystic hemorrhage, or cyst rupture (4). However, we would herein present a case of MCN-L presenting with significant fluid in abdominal and pelvic cavity, intra-operatively diagnosed as MCN-L spontaneous rupture and pathologically confirmed to MCN-L postoperatively.

A 62-year-old female patient with past medical history of hypertension presented with upper abdominal distention and pain for 2 months one and a half years ago. The liver function tests were normal, and the serum tumor markers including alpha-fetoprotein (AFP), carcinoembryonic antigen (CEA), carbohydrate antigen (CA19-9) were all within the normal range. The abdominal contrasted computed tomography (CT) scan demonstrated a $17.9 \mathrm{~cm} \times 12.1 \mathrm{~cm}$ cystic lesion of the liver with smooth and unenhanced edge (Figure 1A). The patient was considered to present with a huge hepatic cyst and underwent laparoscopic fenestration of the hepatic cyst. However, postoperative histopathology suggested low-grade mucinous cystic neoplasm (Figure 1B). The patient was recommended elective surgery for complete lesion resection. But the patient refused to undergo surgery again, and was willing to undergo regular review and close follow-up. At the 1, 6, 12 -month follow-up, the patient was always asymptomatic with negative test results.
The patient suffered from upper abdominal distention and pain again for 1 month recently. The patient went to the emergency department for help because the pain exacerbated and spread to the lower abdomen several days ago. Laboratory tests demonstrated mildly elevated alanine aminotransferase (78 U/L: normal range 9-50), significantly elevated serum CEA (223.1 ng/mL: 0-5) and CA19-9 (>12,000 U/mL: 0-37). The contrasted CT scan and magnetic resonance (MR) demonstrated a $8.8 \mathrm{~cm} \times 6.1 \mathrm{~cm}$ irregular multiloculated cystic lesion within segment IV of the liver with internal septations which enhanced with contrast, presence of irregular wall thickening and mural solid nodules, and significant fluid in abdominal and pelvic cavity, indicating the possibility of cystadenocarcinoma (Figure 1C,D).

Considering that the patient had significant fluid in abdominal and pelvic cavity and might have a malignant tumor with seedling metastases, percutaneous puncture and drainage of peritoneal effusion was performed followed by the biopsy of cystic lesion of the liver. The sample of peritoneal effusion was showed in Figure $1 E$ with slightly brownish color and slightly muddy character, and CEA and CA19-9 in the fluid were both extremely elevated (CEA $>15,000 \mathrm{ng} / \mathrm{mL}$, CA19-9 >12,000 U/mL). Unlike what was expected, the cytology test of peritoneal effusion was negative, the biopsy of cystic lesion of the liver revealed MCN-L with mild dysplasia of glandular epithelium. In order to further eliminate the possibility of false negative biopsy and cytology results, the patient was recommended a positron emission computed tomography (PET-CT) and transvaginal color Doppler ultrasound. But there was still 

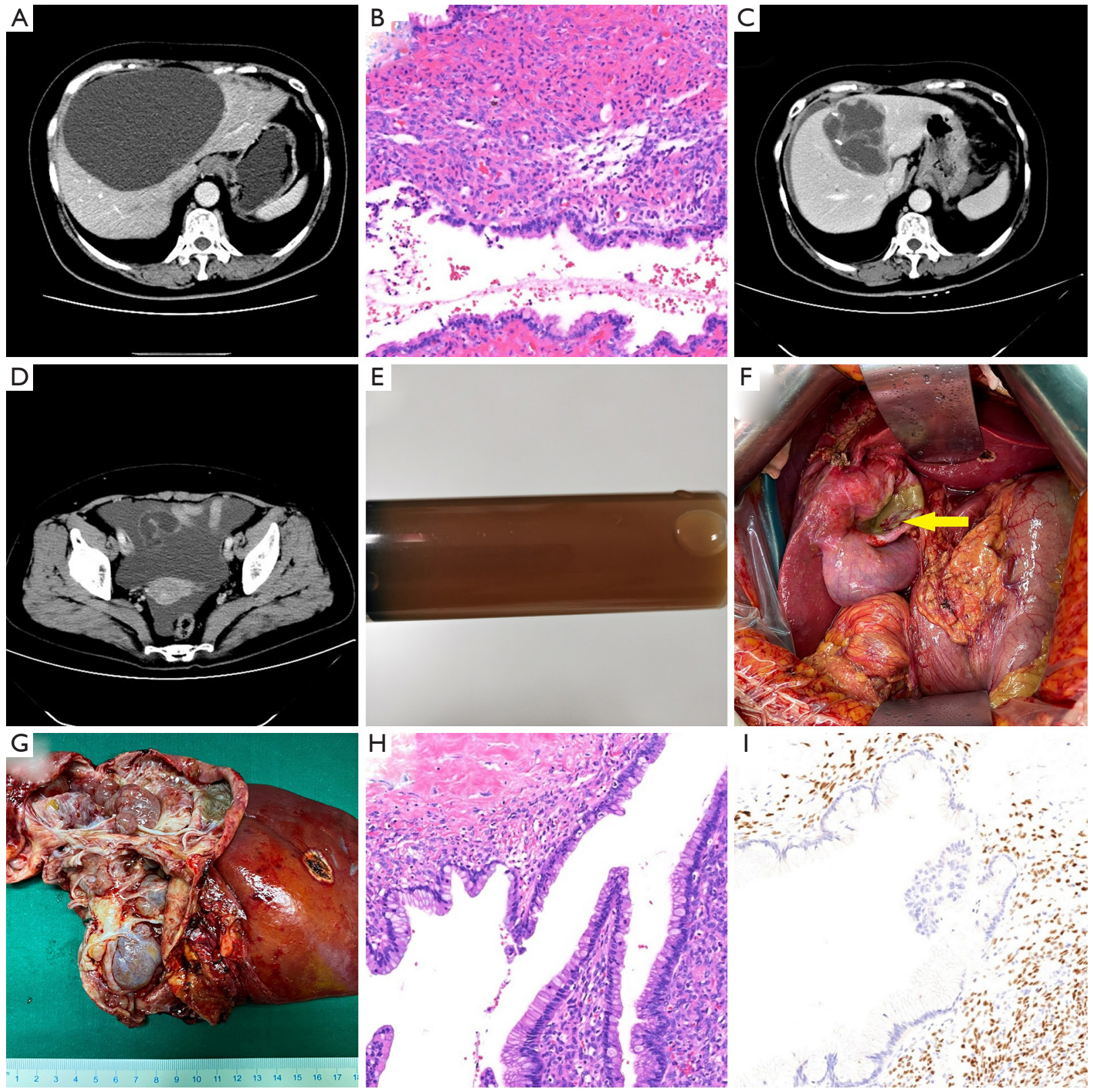

Figure 1 The preoperative imaging, intraoperative photographs, surgical specimen and pathological data (A) The abdominal contrasted CT scan demonstrated a $17.9 \mathrm{~cm} \times 12.1 \mathrm{~cm}$ cystic lesion of the liver with smooth and unenhanced edge on the patient's first admission one and a half years ago. (B) Postoperative histopathology of the hepatic cyst wall suggested low-grade mucinous cystic neoplasm on the patient's first admission one and a half years ago. (C) On the patient's second admission, the contrasted CT scan demonstrated an $8.8 \mathrm{~cm} \times 6.1 \mathrm{~cm}$ irregular multiloculated cystic lesion within segment IV of the liver with internal septations which enhanced with contrast and presence of irregular wall thickening and mural solid nodules, and perihepatic effusion and fluid in the space between the spleen and stomach. (D) CT scan demonstrated significant fluid in the pelvic cavity. (E) The sample of peritoneal effusion with slightly brownish color and slightly muddy character. (F) View of the lesion (the rupture site was shown by the arrow). (G) Resected specimen showing irregular wall thickening and mural solid nodules. $(\mathrm{H})$ Postoperative histology showed the cysts were lined by columnar or cuboidal epithelium with intermediategrade neoplasia and presence of dense subepithelial ovarian-like stroma (hematoxylin-eosin staining). (I) Immunohistochemistry showed that the stroma was positive for estrogen and progesterone receptors. Magnification: 100x. 
no evidence of metastases.

After multidisciplinary discussion, the patient was considered to present with a resectable complex cystic lesion. Intraoperative exploration revealed a huge cystic lesion of the left liver with spontaneous rupture. The rupture site was shown by the arrow in Figure $1 F$. Anatomic left hepatectomy was performed after confirming that there is no obvious abdominal and pelvic metastasis. Irregular wall thickening and mural solid nodules could be found in the resected specimen (Figure $1 G$ ). Intraoperative frozen section examination revealed MCN-L of the left liver with negative resection margin. The patient recovered without any complications, the results of serum CEA and CA19-9 both returned to normal on the 7th day after the surgery, and the patient was discharged on the 10th day after the surgery. A definitive histology results (Figure 1H,I) confirmed MCN-L with intermediate-grade intraepithelial neoplasia.

MCN-L are rare cyst-forming epithelial neoplasm of the liver with slow-growth, most of which are benign, but have potential for malignant transformation. Previous published studies all showed MCN-L predominantly occur in females, especially in the middle-aged females (5-7). The patients with small lesions are usually asymptomatic, while the patients with large lesions may present with abdominal pain or mass, but rarely present with obstructive jaundice, cholangitis, intra-cystic hemorrhage, or cyst rupture (4). Preoperative diagnosis of MCN-L is difficult and sometimes they are quite easily mistaken for hepatic simple cysts (8). It is widely recognized by radiologists that presence of irregular wall thickening, thick calcification, mural solid nodules and papillary projections on CT or MR are suggestive of malignant transformation. Characteristic pathological features of MCN-L are presence of typical mucin-secreting biliary-type columnar or cuboidal epithelium and presence of dense subepithelial ovarian-like stroma with spindle cells expressing female sex hormone receptors $(1,6)$. Complete excision is the treatment of the best choice for MCN-L because of the malignant potential and risk of recurrence (9).

The patient in our case had a huge cystic lesion of the liver with smooth and unenhanced edge in the CT images one and a half years ago, and was misdiagnosed as a huge hepatic cyst and underwent laparoscopic fenestration that was not a very appropriate treatment. The patient presented with significant fluid in abdominal and pelvic cavity for the second time, and was highly suspected of having cystadenocarcinoma because CEA and CA19-
9 in the serum and fluid were all elevated in addition to radiological findings of irregular wall thickening and mural solid nodules. Fortunately, intraoperative exploration and postoperative pathology confirmed no malignant transformation and tumor metastases. The patient was radically cured with a good prognosis after a complete resection with negative resection margin. Spontaneous rupture of MCN-L was a completely unexpected discovery that has never been reported before. Thus, this is an interesting case of MCN-L in which the patient had no typical radiological features at first and then had radiological features of malignant transformation, but in fact there was no malignant change. Besides, spontaneous rupture of MCN-L is theoretically possible, and it does exist.

\section{Acknowledgments}

Funding: None.

\section{Footnote}

Provenance and Peer Review: This article was a standard submission to the Hepatobiliary Surgery and Nutrition. The article did not undergo external peer review.

Conflicts of Interest: All authors have completed the ICMJE uniform disclosure form (available at https://hbsn. amegroups.com/article/view/10.21037/hbsn-21-102/coif). The authors have no conflicts of interest to declare.

Ethical Statement: The authors are accountable for all aspects of the work in ensuring that questions related to the accuracy or integrity of any part of the work are appropriately investigated and resolved. Written informed consent was obtained from the patient for publication of this manuscript and any accompanying images.

Open Access Statement: This is an Open Access article distributed in accordance with the Creative Commons Attribution-NonCommercial-NoDerivs 4.0 International License (CC BY-NC-ND 4.0), which permits the noncommercial replication and distribution of the article with the strict proviso that no changes or edits are made and the original work is properly cited (including links to both the formal publication through the relevant DOI and the license). See: https://creativecommons.org/licenses/by-nc-nd/4.0/. 


\section{References}

1. Nagtegaal ID, Odze RD, Klimstra D, et al. The 2019 WHO classification of tumours of the digestive system. Histopathology 2020;76:182-8.

2. T sui WMS, Adsay NV, Crawford JM, et al. Mucinous cystic neoplasms of the liver. In: Bosman FT, Carneiro F, Hruban RH, Theise ND, eds. World Health Organization classification of tumors. pathology and genetics of tumors of the digestive system. Lyon: IARC Press 2010:IARC Press-8.

3. Kishida N, Shinoda M, Masugi Y, et al. Cystic tumor of the liver without ovarian-like stroma or bile duct communication: two case reports and a review of the literature. World J Surg Oncol 2014;12:229.

4. Kunovsky L, Kala Z, Svaton R, et al. Mucinous cystic neoplasm of the liver or intraductal papillary mucinous neoplasm of the bile duct? A case report and a review of literature. Ann Hepatol 2018;17:519-24.

5. Kubota K, Hachiya H, Nakanuma Y, et al.

Cite this article as: $\mathrm{Xu} \mathrm{X}$, Peng $\mathrm{C}$, Tong R, Dong M, Deng J. An extremely rare phenomenon of mucinous cystic neoplasm of the liver: spontaneous rupture. HepatoBiliary Surg Nutr 2021;10(3):424-427. doi: 10.21037/hbsn-21-102
Clinicopathological features and prognosis of mucinproducing bile duct tumor and mucinous cystic tumor of the liver: A multi-institutional study by the Japan Biliary Association. J Hepatobiliary Pancreat Sci 2014;21:176-85.

6. Zen Y, Pedica F, Patcha V, et al. Mucinous cystic neoplasms of the liver: a clinicopathological study and comparison with intraductal papillary neoplasms of the bile duct. Mod Pathol 2011;24:1079-89.

7. Zen Y, Jang KT, Ahn S, et al. Intraductal papillary neoplasms and mucinous cystic neoplasms of the hepatobiliary system: demographic differences between Asian and Western populations, and comparison with pancreatic counterparts. Histopathology 2014;65:164-73.

8. Anand S, Chandrasekar S, Raja K, et al. Mucinous cystic neoplasm of the liver with biliary communication: an exception to the current classification. BMJ Case Rep 2019;12:bcr-2018-227063.

9. Delis SG, Touloumis Z, Bakoyiannis A, et al. Intrahepatic biliary cystadenoma: a need for radical resection. Eur J Gastroenterol Hepatol 2008;20:10-4. 